\title{
Glassy Mean-Field Dynamics of the Backgammon model
}

\author{
Silvio Franz $\left(^{*}\right)$ and Felix Ritort(**) \\ (*) NORDITA and CONNECT \\ Blegdamsvej 17, \\ DK-2100 Copenhagen Ø(Denmark) \\ e-mail: franz@nordita.dk \\ (**) Departamento de Matematicas, \\ Universidad Carlos III, Butarque 15 \\ Leganés 28911, Madrid (Spain) \\ e-mail: ritort@dulcinea.uc3m.es
}

(August 1995)

\begin{abstract}
In this paper we present an exact study of the relaxation dynamics of the backgammon model. This is a model of a gas of particles in a discrete space which presents glassy phenomena as a result of entropy barriers in configuration space.

The model is simple enough to allow for a complete analytical treatment of the dynamics in infinite dimensions. We first derive a closed equation describing the evolution of the occupation number probabilities, then we generalize the analysis to the study the autocorrelation function.
\end{abstract}

We also consider possible variants of the model which allow to study the effect of energy barriers. 


\section{INTRODUCTION}

The nature of the glass transition is still poorly understood [1,2]. Under slow cooling real glasses reach a metastable phase of free energy larger than that of the crystal phase. Glasses show a strong slowing down of the dynamics when the temperature is lowered and the transport coefficients increase by several orders of magnitude in a narrow range of temperatures. It is natural to think that the appareance of high free-energy barriers is the mechanism responsible for the glass transition. But free-energy barriers are composed of energy barriers and entropy barriers. The question about the relevance of both kind of barriers in real glasses is of the outmost importance. Activated jumps of energy-barriers are strongly dependent on temperature. The typical time $\tau$ to overcome an energy barrier $\Delta E$ is $\tau \sim \exp \left(\frac{\Delta E}{T}\right)$ where $T$ is the temperature. This typical time diverges when the temperature $T$ goes to zero. Conversely, relaxation times related to entropy barriers do not depend directly on the temperature.

The simplest way to visualize entropy barriers is the following, Consider a dynamics in which at each time step the system can reach a new state with uniform probability; the typical time to decrease the energy of one unity is $\tau \sim \frac{\Omega_{i}}{\Omega_{f}}=\exp (\Delta S)$ where $\Delta S$ is the height of the entropy barrier $\left(\Omega_{i}\right.$ stands for the initial available volume of phase space and $\Omega_{f}$ stands for the final volume of phase space with lower energy).

If the effect of energy and entropy barriers is combined one expects that entropy barriers should affect the temperature activated relaxation time in its prefactor $\tau \sim \frac{\Omega_{i}}{\Omega_{f}} \exp \left(\frac{\Delta E}{T}\right)$. According to that, the relaxation time can diverge independently of the temperature if the phase space volume of lower energy configurations in the system shrinks to zero during the dynamical evolution. The idea that an entropy crisis could be relevant to the glassy transition is very old [3, 4], and it has had interesting developements in recent times [5,6] in the framework of mean-field theory of disordered systems. However in the models studied in [5.6] it is very difficult to disentangle entropic effects from energetic ones. To this aim a simple model (the backgammon -BG- model) was recently proposed by one of us [8] (here- 
after referred as I), in which energy barriers are completely absent (a diffusive model with entropy barriers has also been considered in [10]). While the model has no thermodynamic transition, it shows a slow dynamics with strong hysteresis effects and Arrhenius behavior of the relaxation time. The off-equilibrium dynamics of this model was studied subsequently by us [9] (hereafter referred as II) using an adiabatic approximation, obtaining fair good results concerning the relaxation of the energy. The same approximation has been recently rederived, and slightly refined, in a paper by Bouchaud, Godreche and Mézard [11.

In this paper we derive the exact mean-field equation for the order parameter for the dynamics of the BG model, which turns out to be the energy itself. The techniques we use are similar to these of [11], however, the equations we get were not discussed there. We find that the energy verifies a causal functional equation with memory. This is at variance with the approximate treatments where the evolution is described by a Markovian equation.

In its original formulation, the model does not have any energy barriers. However, in real systems energy barriers are present. The BG model is flexible enough to allow for the introduction (and the tuning) of energy barriers. This is done by simple modifications of the Hamiltonian of the system. The formalism developed for the original model applies in these cases.

In the second section we define the Hamiltonian of the BG model and the Monte Carlo dynamics we have used to study it. In the third section we present some exact results for the behavior of the one-time quantities (for instance, the energy) and for the two-time quantities like the correlation function. The fourth section is devoted to the study of the effect of energy barriers in the BG model. Finally we present the conclusions and a discussion of the results.

\section{THE BG MODEL AND THE DYNAMICS}

Let us take $N$ distinguishable particles which can occupy $M$ different states and let us

denote by $\rho=\frac{N}{M}$ the density, i.e. the number of particles per state. The BG Hamiltonian is defined by, 


$$
H=-\sum_{r=1}^{M} \delta_{n_{r}, 0}
$$

where $n_{r}$ is the occupation level of the state $r=1, \ldots, M$, i.e. the number of particles which occupy that state. The numbers $n_{r}$ satisfy the global constraint,

$$
\sum_{r=1}^{M} n_{r}=N
$$

Eq.(1) shows that energy is simply given by the number of empty states (with negative sign). We define the occupation probabilities,

$$
P_{k}=\frac{1}{M} \sum_{r=1}^{M}<\delta_{n_{r}, k}>
$$

which is the probability of finding one state occupied by $k$ particles. The statics of this model in the canonical ensemble can be easily solved (see (I) and (II)). In particular one gets the result,

$$
P_{k}=\rho \frac{z^{k-1} \exp \left(\beta \delta_{k, 0}\right)}{k ! \exp (z)}
$$

where $z$ is the fugacity and $\beta$ is the inverse of the temperature $T$ and they are related by the condition,

$$
\rho\left(e^{\beta}-1\right)=(z-\rho) e^{z}
$$

expressing that the density is fixed to $\rho$.

The probabilities $P_{k}$ satisfy the relation $\sum_{k=0}^{\infty} P_{k}=1$ and they yield all the static observables, in particular the energy $U=-P_{0}$. Several dynamical rules, thermalizing to the Boltzmann distribution, can be attached to the model. The simplest choice (I) is the Metropolis single particle dynamics, in which at each sweep a particle is chosen at random and it is proposed a move to a new state. The move is accepted with probability one if the energy does not increase and with probability $\exp (-\beta)$ otherwise.

In the mean-field version of the model, the possible arrival states of the particles are chosen at random with uniform probability in all the space. This random motion of the 
particles allows a complete analytical treatment of the problem円. Finite dimensional models, where at each sweep the particles are only allowed to move to neighbours on a lattice are currently under study [7].

The model has no energy barriers. Consequently there is no frustration (in the usual sense) and no metastable states. However it was shown in (I) that the dynamics is highly non trivial and a dramatic slowing down occurs at low temperatures. This can be qualitatively understood as follows. Suppose the system is at zero temperature and the dynamics starts from a random initial configuration of high energy. As the system evolves towards the equilibrium more and more states are progressively emptied and the energy decreases. Because the average number of particles per occupied state increases with time (the total number of particles is conserved) the time needed to empty one more state also increases. The result is that the energy goes extremely slowly to its equilibrium value.

The dynamical quantities we are interested in are the time-dependent occupation number probabilities

$$
P_{k}(t)=\frac{1}{M} \sum_{r=1}^{M}\left\langle\delta_{n_{r}(t), k}\right\rangle
$$

$\left(E(t)=-P_{0}(t)\right)$ and the two time energy-energy correlation function [8],

$$
\begin{aligned}
C_{E}(t, s) & =\frac{\frac{1}{M} \sum_{r} \delta_{n_{r}(t), 0} \delta_{n_{r}(s), 0}-E(t) E(s)}{-E(s)(1+E(s))} \\
& \equiv \frac{P\left(n_{r}(t)=0, n_{r}(s)=0\right)-P_{0}(t) P_{0}(s)}{P_{0}(s)\left[1-P_{0}(s)\right]} t \geq s
\end{aligned}
$$

At finite temperature, when $t, s>>t_{e q} \sim \exp (\beta) / \beta^{2}$ (see reference (II) and also section III.B) this function is time translationally invariant. In the regime in which both times $t, s$ are much less than $t_{e q}$, and at all times at zero temperature, the system is off-equilibrium, time-translation invariance does not hold, and the correlation function displays aging (see (8).

\footnotetext{
${ }^{1}$ The interesting case of a sequential dynamics is more complicated.
} 


\section{MEAN-FIELD EQUATIONS FOR THE DYNAMICS OF THE BG MODEL}

In this section we derive exact mean-field equations for the Monte Carlo dynamics of the BG model. First we adress the dynamical problem associated to the one-time probability distributions $P_{k}(t)$. These probabilities generate an infinite hierarchy of Markovian equations which can be closed in terms of the only quantity $P_{0}(t)$. Then we will study the twotime correlation functions in a similar way. For simplicity, we will restrict all the future computations to the case $\rho=1$ (i.e $M=N$ ), the generalization to an arbitrary density being very simple.

\section{A. Dynamical equations for $P_{k}(t)$}

The purpose of this section is to write the dynamical evolution equations for the probabilities $P_{k}(t)$ and, in particular, for the internal energy $E(t)=-P_{0}(t)$. An elementary Monte Carlo move consists in a random selection of one particle (hence, the probability to select a particular departure state $d$ is $n_{d} / N$ where $n_{d}$ is the occupation level of that state) and moving it to a randomly selected arrival state $a$ with uniform probability independent of the occupation level $n_{a}$. One Monte carlo step (our unity of time) consists of $N$ elementary moves. In the elementary move there are several processes which contribute to the variation of $P_{k}(t)$. In appendix A we write explicitly the balance equations, the result is:

$$
\frac{\mathrm{d} P_{k}(t)}{\mathrm{d} t}=(k+1)\left(P_{k+1}-P_{k}\right)+P_{k-1}+P_{0}\left(e^{-\beta}-1\right)\left(\delta_{k, 1}-\delta_{k, 0}-k P_{k}+(k+1) P_{k+1}\right)
$$

where the time index for the probabilities $P_{k}$ has been omitted. The previous equation holds for $k \geq 0$ with $P_{-1}=0$. In particular for $k=0$ we obtain the equation studied in (II),

$$
\frac{\partial P_{0}}{\partial t}=P_{1}\left(1-P_{0}\right)-e^{-\beta} P_{0}\left(1-P_{1}\right)
$$

The hierarchy was closed in (II) assuming fast relaxation on the surfaces of constant energy, and slow variation of the energy itself. In this condition eq.(9) was solved assuming $P_{k}(t)=$ $\exp \left[\beta(t) \delta_{k, 0}-z(t)\right] \frac{z(t)^{k-1}}{k !}$ with $\beta(t)$ and $z(t)$ related at all times by eq.(5). 
Here we study the hyerarchy (9) with the method of the generating function, that we define as

$$
G(x, t)=\sum_{k=0}^{\infty} x^{k} P_{k}(t)
$$

A similar approach was also used in [11] where the adiabatic approximation of (II) was rederived and improved[2].

From the equation (8) it is easy to check that the $G(x, t)$ satisfies the partial differential equation,

$$
\frac{\partial G(x, t)}{\partial t}=(x-1)\left[G(x, t)+\lambda(t)-(1+\lambda(t)) \frac{\partial G(x, t)}{\partial x}\right]
$$

with $\lambda(t)=P_{0}(t)\left(e^{-\beta}-1\right)$. Eq. (11) is a non linear partial differential equation, the nonlinearity is contained in the dependence of $\lambda$ on $P_{0}(t)=G(0, t)$.

The equilibrium solution $G_{e q}(x)$ is easily obtained from equations (4) and (10),

$$
G_{e q}(x)=\frac{e^{\beta}-1+e^{z x}}{z e^{z}}
$$

and one can check that this is consistent with eq.(11).

The previous partial differential equation can be implicitly solved to get $G(x, t)$ as a functional of $\lambda$. The details are presented in the Appendix B, we give here the result

$$
G(x, t)=\mathrm{e}^{(x-1) D(t, 0)} G_{0}(1+(x-1) B(t, 0))+(x-1) \int_{0}^{t} \mathrm{~d} s \lambda(s) B(t, s) \mathrm{e}^{(x-1) D(t, s)}
$$

where we have written

$$
\begin{aligned}
& B(t, s)=\exp \left(-\int_{s}^{t} \mathrm{~d} v[1+\lambda(v)]\right) \\
& D(t, s)=\int_{s}^{t} \mathrm{~d} v B(t, v)
\end{aligned}
$$

and $G_{0}(x)=G(x, 0)$ is the initial condition at time $t=0$. Setting $x=0$ in (13) we get a closed equation for $P_{0}(t)$ which reads

\footnotetext{
${ }^{2}$ The technique of the generating function in the study of the dynamics has also been applied to some mean-field spin glass models [12].
} 


$$
P_{0}(t)=\mathrm{e}^{-D(t, 0)} G_{0}(1-B(t, 0))+\left(1-e^{-\beta}\right) \int_{0}^{t} \mathrm{~d} s P_{0}(s) B(t, s) \mathrm{e}^{D(t, s)}
$$

The previous equation, although strongly non-markovian is causal, as the l.h.s. depends on the values of $P_{0}(s)$ for $s \leq t$. It has a unique solution that can be found numerically with good precision, discretizing the time and integrating it step by step. The evaluation of the previous expressions gives the full solution of the BG model as far as the one-time dynamical quantities are concerned.

The solution of (13) is explicit at infinite temperature $(\beta=0)$. In this case $\lambda(t)=0$ and the solution of $G(x, t)$ simplifies,

$$
G(x, t)=e^{\left(1-e^{-t}\right)(x-1)} G_{0}\left((x-1) e^{-t}+1\right)
$$

It is not surprising that at infinite temperature the system goes exponentially fast to the equilibrium (with relaxation time equal to 1). At infinite temperature the equilibrium probabilities eq.(四) are given by $P_{k}=\frac{1}{k ! e}$, the energy being $E=-P_{0}=-\frac{1}{e}$. If we start from the initial condition in which all particles occupy the same state $\left(P_{0}=1, P_{k}=0, k>0\right)$ then we have $G_{0}(x)=1$. From eq.(16) we obtain the time evolution of the energy,

$$
E(t)=-G(0, t)=-e^{e^{-t}-1}
$$

We studied numerically the solution of (15) at $T=0$. In figure 1 we display the result for the energy, starting from the initial condition $P_{k}(0)=1 /(e k !)$ at time 0 (i.e. $\left.G_{0}(x)=e^{x-1}\right)$. For comparison we plot the results of the Monte Carlo simulations and of the adiabatic hypothesis of (II) with the same initial condition.?

\section{B. The Correlation Function $C_{E}(t, s)$}

In this section we investigate the behavior of the energy-energy correlation functions (7). We proceed in a similar way as we have done for the occupation probabilities. We need to

\footnotetext{
${ }^{3}$ The adiabatic hypothesis gives better results if the integration is started at latter times.
} 
study the joint occupation probability in a given site $r$ at two different times $t, s(t>s)$, $P\left(n_{r}(t)=0, n_{r}(s)=0\right)=P\left(n_{r}(t)=0 \mid n_{r}(s)=0\right) P_{0}(s)$. The correlation function eq. (7) can be written as

$$
C_{E}(t, s)=\frac{P\left(n_{r}(t)=0 \mid n_{r}(s)=0\right)-P_{0}(t)}{1-P_{0}(s)}
$$

We now write a set of equations that allow to study $P\left(n_{r}(t)=0 \mid n_{r}(s)=0\right)$.

Let us define the probabilities

$$
\nu_{k}(t, s)=P\left(n_{r}(t)=k \mid n_{r}(s)=0\right)
$$

i.e. the occupation number probabilities in the set $S_{s}$ of states which are empty at time $s$. In general, it is possible to restrict the balance equations that led to (8) to any subset $S$ of the whole space. Irrespectively of $S$ the result is:

$$
\begin{aligned}
\frac{\partial \nu_{k}}{\partial t}= & \nu_{k-1}-\nu_{k}+\left[(k+1) \nu_{k+1}-k \nu_{k}\right]\left[1-P_{0}\left(1-e^{-\beta}\right)\right] \\
& -\left(\delta_{k, 1}-\delta_{k, 0}\right)\left[\nu_{0}\left(1-P_{1}\right)+\nu_{1} P_{0}\right]\left(1-e^{-\beta}\right)
\end{aligned}
$$

In particular if the set $S$ is the whole space $\nu_{k}=P_{k}$ and we get back to (8).

Of course the initial conditions depend on the set under study. For the set $S_{s}$ we are interested to, we must choose

$$
\nu_{k}(s, s)=\delta_{k, 0}
$$

In terms of the generating function

$$
\Gamma(x, t, s)=\sum_{k=0}^{\infty} x^{k} \nu_{k}(t, s)
$$

eq.(20) reads

$$
\frac{\partial \Gamma}{\partial t}=(x-1)\left[\Gamma-\left(1-P_{0}\left(1-e^{-\beta}\right)\right) \frac{\partial \Gamma}{\partial x}-\left(\nu_{0}\left(1-P_{1}\right)+\nu_{1} P_{0}\right)\left(1-e^{-\beta}\right)\right]
$$

with condition at time $s$

$$
\Gamma_{s}(x) \equiv \Gamma(x, s, s)=1 \text {. }
$$


Note that if we suppose to know the $P_{k}(t)$ then the eq. (20,23) are linear. Obviously, if one considers the set $\bar{S}_{s}$ complementary to $S_{s}$, and its respective generating function $\bar{\Gamma}$, it holds the equality: $P_{0}(s) \Gamma(x, t, s)+\left(1-P_{0}(s)\right) \bar{\Gamma}(x, t, s)=G(x, t, s)$.

Defining

$$
\begin{aligned}
& \mu(t, s)=\left[\nu_{0}(t, s)\left(1-P_{1}(t)\right)+\nu_{1}(t, s) P_{0}(t)\right]\left(1-\mathrm{e}^{-\beta}\right) \\
& B(t, s)=\exp \left(-\int_{s}^{t} \mathrm{~d} v\left(1-P_{0}(t)\left(1-\mathrm{e}^{-\beta}\right)\right)\right) \\
& D(t, s)=\int_{s}^{t} \mathrm{~d} v B(t, v)
\end{aligned}
$$

we find

$$
\Gamma(x, t, s)=\mathrm{e}^{(x-1) D(t, s)}-(x-1) \int_{s}^{t} \mathrm{~d} u \mu(u, s) B(t, u) \mathrm{e}^{(x-1) D(t, u)}
$$

which depends implicitly on $\nu_{0}$ and $\nu_{1}$. In order to find a closed system we have to consider eq.(26) and its $x$-derivative in $x=0$

$$
\begin{aligned}
& \nu_{0}(t, s)=1+\int_{s}^{t} \mathrm{~d} u\left[-\nu_{0}(u, s)\left[1-\left(1-P_{1}(u)\right)\left(1-\mathrm{e}^{-\beta}\right)\right]+\nu_{1}(u, s)\right] \\
& \nu_{1}(t, s)=\int_{s}^{t} \mathrm{~d} u\left[\mu(u, s) B(t, u) \mathrm{e}^{-D(t, u)}(D(t, u)-1)\right]+\mathrm{e}^{-D(t, s)} D(t, s)
\end{aligned}
$$

The system (27), if one assumes known the probabilities $P_{k}(t)$, consists in a vectorial linear Volterra equation of second kind for $\nu_{0}$ and $\nu_{1}$ which can in all generality be integrated numerically, and in some particular case also analytically.

The simplest case is the equilibrium at finite temperature. In that case, $P_{k} \equiv P_{k}^{e q}$ and the various functions appearing in (27) are time traslation invariant. In these conditions eq.(27) can be solved in Laplace transform. Simple algebra, and the formula (see e.g. [13])

$$
\int_{0}^{\infty} d t \exp (-a \exp (t)-E)=a^{-E} \gamma(p, a)
$$

( $\gamma$ is the incomplete gamma function), shows that $\nu_{0}(E)$, the Laplace transform of $\nu_{0}(t-s)$, is given by

$$
\nu_{0}(E)=\frac{A(E)+\frac{z-1}{z}(1-E A(E))}{1-\left[\frac{(z-1) e^{z}}{(z-1) e^{z}+1}+\frac{z-1}{z} E\right](1-E A(E))}
$$


where we have expressed all the equilibrium quantities in terms of the fugacity $z$ (see eq. (5)), $E$ is the Laplace variable conjugated to time, and

$$
A(E)=\frac{1}{e^{z} z^{z E-1}} \int_{0}^{z} u^{z E-1} e^{u} .
$$

$\nu_{0}(E)$, as it should, has a pole in $E=0$ with residue $P_{0}$ coresponding to $\nu_{0}(t) \rightarrow P_{0}$ for large time. Poles on the real negative $E$ axis correspond to exponential relaxation modes. The largest relaxation time is given by minus the inverse of the value of $E$ in the pole closest to the origin. This can be obtained explicitely for large $\beta$, where $z \approx \beta-\log (\beta)$ is large, from the asympotic expantion of $E A(E)$ for small $E$

$$
E A(E) \approx e^{-z}+E .
$$

The result is simply $E_{\text {pole }} \approx-e^{-z}$ and correspondingly $\tau_{\max } \approx e^{z} \approx \exp (\beta) / \beta$.

In the off-equilibrium regime the integration of (27) can be performed numerically. In fig. 2 we show the result of the integration for $T=0$ for different values of $s$ (i.e. different waiting times) compared to the Monte Carlo results. Although we did not try very sophisticated algorithms, with standard ones [14], we were able to reach enough large times to see the scaling behavior $C_{E}(t, s)=f((t-s) / s)$ observed numerically in I. It would be interesting to see if equation (26) could be solved with the aid of some simple approximation as it is the case for the energy (II and [11]).

\section{THE EFFECT OF ENERGY BARRIERS}

The BG model has no energy barriers and hence there is no finite temperature thermodynamic phase transition. In real glasses energy barriers are always present and it can be instructive to understand their effect when combined with entropy barriers. One can easily modify the Hamiltonian (1D) to include energy barriers. In this paper we have focused on two different ways. In the first, we have considered interaction between the different states, introducing an energy gain when groups of states are simultaneously empty. This interaction term is enough to make appear a finite temperature thermodynamic transition, but 
metastability and frustration are absent and the system monotonically reaches the ground state at zero temperature. In the other, we introduced metastable configurations in the dynamics. In this case the system fails to reach the ground state at zero temperature while thermodinamically there is no finite-temperature phase transition.

\section{A. The $p$-states model}

The simplest way we can introduce interaction among different states in the model is the following, consider the quantity

$$
M\left[\left\{n_{r}\right\}\right]=\frac{1}{N} \sum_{r=1}^{N}\left(\delta_{n_{r}, 0}-1 / e\right) .
$$

Any Hamiltonian of the form

$$
H=N F\left(M\left[\left\{n_{r}\right\}\right]\right)
$$

with $F$ gentle enough, is a good candidate for a mean-field model. We did not try a systematic study of the form (33) for generic $F$, but we concentrated to the class of monomials, where

$$
H_{p}=-\frac{1}{N^{p-1}}\left(\sum_{r=1}^{N}\left(\delta_{n_{r}, 0}-1 / e\right)\right)^{p}
$$

For $p=1$ this model reduces to the BG model. For larger values of $p$ there is interaction between different states. The ground state of this model is the same as the one of the BG model (all particles occupying the same state) and there are no energy barriers at zero temperature. A careful study of the thermodynamics of this model shows that for any $p>1$ there is a first order phase transition from a completely disordered phase with $M=0$ for $T>T_{c}$ to an 'ordered' phase with $M \neq 0$ for $T<T_{c}$. This leads to the curious situation that the completely disordered state is dynamically stable at all temperatures but at $T=0$. This can be understood by a simple argument. Suppose to start the dynamics in a random initial condition and consider a sweep that lead to the filling of an empty state. The energy variation in this process is 


$$
\delta H=-\frac{1}{N^{p-1}}\left[\left(\sum_{r}\left(\delta_{n_{r}, 0}-\frac{1}{e}\right)-1\right)^{p}-\left(\sum_{r}\left(\delta_{n_{r}, 0}-\frac{1}{e}\right)\right)^{p}\right] \simeq \frac{1}{N^{p-1}} p\left(\sum_{r}\left(\delta_{n_{r}, 0}-\frac{1}{e}\right)\right)^{p-1} .
$$

But according to our hypothesis $\sum_{r}\left(\delta_{n_{r}, 0}-\frac{1}{e}\right)$ is a random variable of order $\sqrt{N}$, correspondingly $\delta H \sim N^{-(p-1) / 2}$ and the acceptance rate

$$
\mathrm{e}^{-\beta \delta H} \sim \mathrm{e}^{-\beta / N^{(p-1) / 2}}
$$

For finite temperature and large $N$ all the moves are accepted and the energy in average never decreases. In other words the statistic of configurations is not changed by the dynamics. A crossover is found for $\beta \sim N^{(p-1) / 2}$ showing that the relevant scale of temperature for the dynamics is different from that of the statics. Right at zero temperature, where only the sign of the energy change and not the magnitude matters, the dynamics of the model coincides for any $p$ with the one of the conventional case $p=1$.

\section{B. The effect of metastability}

The $p$-states model has no metastability at zero temperature. We want to study here a simple model where metastability is present but without interaction. In the BG model the ground state is reached by emptying progressively more and more states. To empty a given state at a certain time $t$ it is necessary to pass in a configuration where a unique particle occupies that state. We then consider the following model,

$$
H=\sum_{r=1}^{N}\left(-\delta_{n_{r}, 0}+g \delta_{n_{r}, 1}\right)
$$

where $g$ is positive constant and we have the usual constraint eq.(2). At zero temperature the transition $n_{r}=2 \rightarrow n_{r}=1$ is forbidden, hence energy barriers are present in the model. More general models are obtained including in the Hamiltonian all possible terms of the type $\delta_{n_{r}, k}$

$$
H=-\sum_{r=1}^{N} \sum_{k=0}^{\infty} g_{k} \delta_{n_{r}, k}=-\sum_{r=1}^{N} g_{n_{r}}
$$


We focus here to the case (37). The statics of this model is easily solved. We obtain the free energy,

$$
\beta f=\log (z)-\log \left(e^{z}+e^{\beta}-1+z\left(e^{-\beta g}-1\right)\right)
$$

and the fugacity is related to the temperature $\frac{1}{\beta}$ by the $g$-independent relation eq.(5). The equilibrium probabilities $P_{k}$ (see eq.(3)) are given by,

$$
P_{k}=\frac{z^{k-1} \exp \left(\beta \delta_{k, 0}-\beta g \delta_{k, 1}\right)}{k !\left(e^{z}+e^{-\beta g}-1\right)}
$$

The dynamics of this model is expected to be substantially different to that of the BG model at least at very low temperatures. Concretely, at zero temperature the ground state is the same as for the BG case but there is a large number of metastable states (for instance, half states empty and half of the states with two particles). It is easy to show that for each value of $E$ between $E=-1 / 2$ and the ground state $E=-1$ there exists a metastable configuration with that energy. Then we expect the value of the energy extrapolated to infinite time to depend strongly on the initial configuration. In order to minimize the energy we have to maximize $P_{0}$ and minimize $P_{1}$. While the maximization of $P_{0}$ is a process where entropy barriers are dominant (this is the reason why the $\mathrm{BG}$ model defined as $E=-P_{0}$ is interesting) this is not the case for minimizing $P_{1}$ where entropy barriers are absent. Then, independently of the initial configuration we expect that $P_{1}$ will go to zero in the exponentially fast for large times. In these conditions, we do not expect that the adiabatic solution of (II) can give a good approximation of the dynamics. This approximation was based on the fact that in the BG the surfaces of constant energy are connected, a situation which does not hold here.

However the dynamics of this model can be directly solved as in the BG case. Skipping all the details we find for the generating function:

$$
\begin{array}{r}
\frac{\partial G(x, t)}{\partial t}=(x-1)\left(-(1+\lambda(t)) \frac{\partial G(x, t)}{\partial x}+\left(1+2 P_{2}\left(e^{-\beta g}-1\right)\right) G(x, t)+\right. \\
\lambda(t)-2 P_{2}\left(e^{-\beta g}-1\right)\left(e^{x}-P_{0}(x-1)\left(1-e^{-\beta(1+g)}\right)\right)
\end{array}
$$


where $\lambda(t)=P_{0}(t)\left(e^{-\beta(1+g)}-1\right)$. Observe that eq. (41) depends only on the probabilities $P_{0}(t)$ and $P_{2}(t)$. The solution is more complicated to that of eq.(13), however it can be found. In figure 3 we show the result of the numerical integration of (41) for the energy at $T=0$ compared with the Monte Carlo simulations, starting from a completely random initial condition. The energy seems to converge to a value $\lim _{t \rightarrow \infty} E(t)=-.564$, a result that it would be interesting to derive analytically. It is under current study the finite temperature dynamics, where we expect the effect of the energy and entropy barriers to combine to give rise to a dynamics slower than that of the BG model.

\section{CONCLUSIONS}

In this paper we have derived the exact mean-field equations of the dynamics of the Backgammon model. This has been achieved through the study of the single site occupation number probability for which a hierarchical set of equations hold. With the method of the characteristic function, we have derived a closed functional equation for the energy. This, although non-Markovian, has a causal character and can be integrated step by step discretizing the time. The non-Markovian character of the evolution equation, suggests that history dependent effects should be observable in the system. However the analysis of II, where the evolution of the energy was described by an approximate equation, shows that even in subtle phenomena as hysteresis cycles in cooling-heating processes, history dependent effects are very small. This should reflect in the fact that the memory kernels that appear in the equation for the energy are short range in time.

The method of the generating function also allowed us to derive also a system of linear Volterra equations describing the evolution of the energy autocorrelation function. The numerical solution of these equation confirmed the aging behaviour found in I. It would be interesting to derive analytically the scaling $C_{E}\left(t, t^{\prime}\right)=f\left(t^{\prime} / t\right)$.

In the last section we have derived the mean-field theory for a model where entropic and

energetic barriers are combined. We have seen that at temperature zero, starting from a 
random configuration the system fails in finding the ground state. For future work it is left the study of this model for finite temperature.

Non linear equations with memory appear in phenomenological glass theory under the name of Mode Coupling Theory [1]. Mode coupling equations appear naturally in the meanfield treatment of the dynamics of disordered [15, 16] or quasi-disorderd systems [17], in offequilibrium situation they involve a set of coupled integral equation for the two time autocorrelation function and its conjugated response function. The most striking manifestation of the importance of memory effects in off-equilibrium mode coupling theory is in the aging behaviour of the response function [15, 16].

Structural glasses are generally classified as strong glasses (Arrhenius behavior of the relaxation time) or fragile glasses (Vogel-Tamman-Fulcher behavior of the relaxation time). In this classification the BG model is a strong glass. Polymer glasses are fragile glasses which show strong aging effects in its physical properties [2]. It would be desiderable to know from experiments if there is a correlation between the fragility of glasses and its aging properties. This could shed light on the role of energy barriers in the mechanism of the glass transition. We believe that only entropy barriers cannot yield aging effects in the response function. In this framework a more detailed study of the BG model with metastability (as presented in the last section) at finite temperature could be instructive. In particular, the study of the relaxation time as a function of the temperature and the existence of aging due to the presence of energy barriers.

\section{Acknowledgements}

We would like to thank J.P. Bouchaud, E.Follana and M. Mézard for interesting discussions. (F.R.) acknowledges Ministerio de Educacion y Ciencia of Spain for financial support.

\section{APPENDIX A}

In this appendix we derive the evolution equation for the probability $P_{k}(t)$. Define as $N_{k}(t)$ the number of states occupied by $k$ particles $\left(P_{k}(t)=N_{k}(t) / N\right)$. 
The processes leading to a variation of $N_{k}$ can be classified in this way:

- Process $A+$ : arrival of a particle in a state with $k-1$ particles.

- Process $A-$ : departure of a particle from a state with $k$ particles.

- Process $B+$ : departure of a particle from a state with $k+1$ particles.

- Process $B$-: arrival of a particle in a state with $k$ particles.

Note that in a sweep the processes above are not mutually exclusive, so, for example the contemporary occurency of $A+$ and $B$ - lead to no variation in $N_{k}$. At each Monte Carlo sweep three independent random variables are extracted: a departure state $d$ with probability $n_{d} / N$, an arrival state $a$ with probability $1 / N$ and an acceptance variable

$$
x=\left\{\begin{array}{l}
1 \text { with } \text { prob. } \mathrm{e}^{-\beta} \\
0 \text { with prob. } 1-\mathrm{e}^{-\beta}
\end{array}\right.
$$

In terms of these variables the variation in $N_{k}$ in each process is given by:

- Process $A+: \quad \delta_{n_{a}, k-1}\left[1-\delta_{k, 1}\left(1-\delta_{n_{d}, 1}\right) \delta_{x, 0}\right]$

- Process $A-: \quad-\delta_{n_{d}, k}\left[1-\delta_{n_{a}, 0} \delta_{x, 0}+\delta_{k, 1}+\delta_{k, 1} \delta_{n_{a}, 0} \delta_{x, 0}\right]$

- Process $B+: \quad \delta_{n_{d}, k+1}\left[1-\delta_{n_{a}, 0} \delta_{x, 0}+\delta_{k, 0} \delta_{n_{a}, 0} \delta_{x, 0}\right]$

- Process $B-: \quad-\delta_{n_{a}, k}\left[1-\delta_{k, 0}\left(1-\delta_{n_{d}, 1}\right) \delta_{x, 0}\right]$

The contribution in the different processes can be easily understood, for example in process A+ we must have $k-1$ particles in the arrival state. If $k=1$ and $n_{d}>1$ the move imply an energy cost, and is accepted only if $x=1$.

Summing all the contribution and averaging over $p, a$ and $x$ we find:

$$
\begin{aligned}
& \left\langle N_{k}(t+\delta t)-N_{k}(t)\right\rangle=N\left[P_{k}(t+\delta t)-P_{k}(t)\right] \equiv \frac{\mathrm{d} P_{k}(t)}{\mathrm{d} t}= \\
& (k+1)\left(P_{k+1}-P_{k}\right)+P_{k-1}+P_{0}\left(e^{-\beta}-1\right)\left(\delta_{k, 1}-\delta_{k, 0}-k P_{k}+(k+1) P_{k+1}\right)
\end{aligned}
$$

Very similar considerations lead to (20) if one restricts the balance equation to a subset of the whole space. 


\section{APPENDIX B}

In this Appendix we obtain the solution of eq.(11). We perform the change of variables $(x, t) \rightarrow(u, t)$ where $x-1=e^{u+\int_{0}^{t} \mathrm{~d} s(1+\lambda(s))}=e^{u} B(t, 0)$. In terms of the new variables eq.(11) reads,

$$
\frac{\partial \hat{G}}{\partial t}=e^{u} B(t, 0)(\hat{G}+\lambda)
$$

where $\hat{G}(u, t)=G(x(u, t), t)$ This is a linear differential equation which can be readily solved

$$
\hat{G}(u, t)=e^{e^{u} \int_{0}^{t} \mathrm{~d} s B(s, 0)} F(u)+e^{u} \int_{0}^{t} \mathrm{~d} s \lambda(s) B(s, 0) e^{e^{u} \int_{s}^{t} \mathrm{~d} v B(v, 0)}
$$

where $F$ is an arbitrary function.

Going back to $(x, t)$ and imposing the initial condition we get eq.(13). 


\section{REFERENCES}

[1] W. Gotze, Liquid, freezing and the Glass transition, Les Houches (1989), J. P. Hansen, D. Levesque, J. Zinn-Justin editors, North Holland;

[2] C. A. Angell, Formation of Glasses from Liquids and Biopolymers Science, 267 (1995) 1924

[3] W. Kauzmann, Chem. Rev. 43 (1948) 219

[4] G. Adam, J.H. Gibbs, J. Chem. Phys. 43 (1965) 139

[5] T.R. Kirkpatrick and P.G. Wolynes, Phys. Rev. B 36 (1987) 8552; T.R. Kirkpatrick, D. Thirumalai and P.G. Wolynes Phys. Rev. A 40 (1989) 1045.

[6] G. Parisi, Gauge Theory, Spin Glasses and Real Glasses and references therein preprint cond-mat/9411115,

[7] E.Follana and F.Ritort, work in progress.

[8] F. Ritort, Phys. Rev. Lett. 75 (1995) 1190;

[9] S. Franz, F.Ritort, Dynamical solution of a model without energy barriers , Preprint cond-mat 9505115 to appear in Europhys. Lett.

[10] A. Barrat and M. Mezard, Phase-space diffusion and low temperature aging preprint ENS 95/16 to appear in J. Physique

[11] J.P. Bouchaud, C. Godrèche, M. Mézard, Entropy barriers and slow relaxation in some random walk models Preprint.

[12] L. L. Bonilla, F. G. Padilla, G. Parisi and F. Ritort, Analytical solution of the Monte Carlo dynamics of a simple spin-glass model Preprint cond-mat 9508004

[13] H. Bateman, Tables of integral transforms McGraw-Hill New York (1954).

[14] W. H. Press et al. Numerical Recipes, the art of scientific computing 2nd edition, Cam- 
bridge (1992)

[15] L. F. Cugliandolo and J. Kurchan, Phys. Rev. Lett. 71 (1993) 173; J. Phys. A 27 (1994) 5749

[16] S. Franz and M. Mézard, Europhys. Lett. 26 (1994) 209; Physica A210 (1994) 48.

[17] S.Franz and J.Hertz, Phys. Rev. Lett. 74 (1995) 2114. 


\section{Figure Captions}

Fig. 1 The decay of the energy at zero temperature starting from a completely random configuration at time $t=0$. We compare the numerical solution of (15) (full lines) with the Monte Carlo simulations for $N=10^{5}$ and the integration of the adiabatic equation of II with the same initial condition.

Fig. 2 The correlation function at zero temperature as a function of $\left(t-t_{w}\right) / t_{w}$ for different $t_{w}\left(t_{w}=10,30,100,300\right)$. We take it as a good indication for the $t / t_{w}$ scaling at large times.

Fig. 3 The correlation function at zero temperature for $t_{w}=10$ compared with the montecarlo data for $N=10^{5}$.

Fig. 4 Energy vs. time in the model with energy barriers (theory + Monte Carlo data at $N=10^{5}$ ), starting from a random configuration at time zero. We observe exponential decay to $E=-.564$. 